\title{
A importância do processo de alinhamento da estratégia com projetos Seis Sigma: um estudo multicasos em operadores logísticos
}

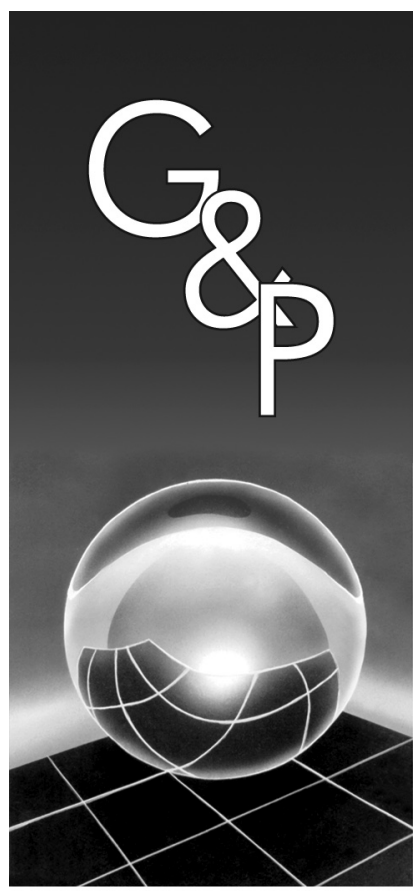

Bianca Soares de Oliveira Gonçalves

Marcel Andreotti Musetti

\begin{abstract}
Resumo
Além do fato do programa Seis Sigma e a logística estarem em evidência no ambiente organizacional, soma-se a importância de se obter sucesso na implantação do programa, caracterizando como um fator crítico o alinhamento da estratégia dos projetos de melhoria. Sendo assim, este artigo propõe a sistematização do alinhamento da estratégia com o processo de seleção de projetos Seis Sigma. Dessa forma, esta pesquisa está estruturada, primeiramente, com uma breve descrição dos modelos conceituais utilizados e sobre operadores logísticos, os quais são os casos desta pesquisa. Com o embasamento teórico, é proposta uma sistematização conceitual do alinhamento da estratégia do processo de seleção de projetos Seis Sigma detalhado em um passo a passo. Com o objetivo de analisar a proposta conceitual, realizaram-se três estudos de casos em operadores logísticos. Podem-se notar a importância do processo de seleção de projetos Seis Sigma e seu alinhamento com a estratégia, bem como a validação da sistematização proposta.
\end{abstract}

Palavras-chave: Projetos Seis Sigma. Estratégia e alinhamento.

\section{Introdução}

As teorias administrativas utilizadas nos tempos atuais invariavelmente citam a necessidade de implantação de melhorias nas empresas. Sejam motivadas pelos clientes, ou pelos competidores, elas são consideradas fundamentais para a sobrevivência da empresa em um ambiente de contínua mudança como o que se apresenta atualmente.

Uma alternativa de melhoria pode surgir de programas que apóiam a competitividade e o desempenho organizacional como o programa Seis Sigma. Segundo Santos e Martins (2005), o programa Seis Sigma vem ganhando força nas organizações como um programa de qualidade que promove a elevação do desempenho organizacional e, por isso, está ficando cada vez mais inserido no plano estratégico das organizações. Segundo Gerolamo (2003), para que as ações de melhoria estejam coerentes com a estratégia é preciso desdobrá-la e estabelecer prioridades de investimentos nas áreas que impulsionem a estratégia pretendida. No Seis Sigma, a priorização pode ser alcançada por meio da seleção de projetos Seis Sigma, pois este processo direciona a força de melhoria (WERKEMA, 2004).

A literatura pesquisada, porém, não apresenta como o alinhamento entre o programa Seis Sigma e a estratégia ocorre em detalhes, sendo este o principal objetivo deste artigo. Assim, este artigo propõe uma sistematização do processo de alinhamento estratégico de projetos Seis Sigma e busca uma validação junto a operadores logísticos. A amostra de empresas se justifica, pois uma das atividades que vem sendo transformada radicalmente é a logística, a qual, de parte acessória e complementar ao processo produtivo, passa a se inserir como fundamental no posicionamento estratégico das empresas, tendo em vista o processo de criação de valor e atendimento às necessidades dos clientes, cada vez mais exigentes em termos de prazos, preços e serviços (BOWERSOX et al., 2001).

Existem vários conceitos para Seis Sigma. Pode ser uma estratégia de negócio que procura identificar e eliminar causas de erros ou defeitos nos processos de negócio, focando as características do produto que são críticas para o consumidor (ANTONY, 2004). Já para Harry et al. (1998), Seis Sigma é um processo de negócio que permite às organizações aumentarem seus lucros por meio da otimização das operações, melhoria da qualidade e eliminação de defeitos. Harry e Crawford (2005) entendem o Seis Sigma como uma ferramenta 
que agrega valor ao produto para o cliente. Para Senapati (2004), o Seis Sigma é um método para melhoramento de processos. Segundo Bisgaard, Hoerl e Snee (2002), Seis Sigma é uma abordagem que visa melhorias no negócio, por meio da eliminação das causas de erros e defeitos nos processos de negócio, com foco nas necessidades do consumidor. Rotandaro (2002) acrescenta que Seis Sigma é uma filosofia de trabalho para alcançar, maximizar e manter o sucesso comercial, por meio da compreensão das necessidades do cliente.

Seguindo a classificação de Gil (1993), a pesquisa realizada foi caracterizada como exploratória, já que busca uma maior familiarização com o problema. A abordagem adotada neste trabalho é qualitativa, pois, para o desenvolvimento do tema, é preciso compreender em profundidade, o contexto que envolve o problema de pesquisa (BRYMAN, 1989). Além disso, as evidências devem ser coletadas com a ajuda de entrevistados internos às empresas pesquisadas. A realização deste trabalho pautou-se pela utilização do método de estudo de casos múltiplos, além de uma revisão bibliográfica. Para isso, foram selecionadas empresas que fossem operadores logísticos e que utilizassem o programa Seis Sigma para otimização da logística. Foram selecionadas três empresas, América Latina Logística (ALL), Companhia Vale do Rio Doce e MasterDis (nome fictício, pois a empresa não autorizou a divulgação de seu nome), por estas atenderem às necessidades específicas para a realização desta pesquisa.

\section{Embasamento teórico: apresentação dos modelos utilizados}

Para o desenvolvimento da proposta de sistematização supracitada, foram utilizadas as propostas dos autores: Gerolamo (2003); Werkema (2004); Kaplan e Norton (1990); Yip (1995); e Santos e Martins (2004).

\subsection{Modelo conceitual para o processo de ges- tão de melhoria e mudança de desempenho}

Esta abordagem propõe um modelo conceitual para o processo de gestão de melhoria e mudança de desempenho. Desdobrando o modelo, são descritos os principais passos existentes nele, ilustrados pela Figura 1, que podem ser divididos em três processos-chave: revisar; formular e atualizar a estratégia; desdobrar ações de melhoria e mudança; e avaliar e medir o desempenho organizacional. Este modelo tem como principal objetivo direcionar a força de melhoria para os objetivos estratégicos da empresa.

O primeiro passo é analisar os aspectos-chave que são levados em consideração para se analisar uma estratégia, a fim de que a empresa não seja surpreendida por eventuais ameaças que coloquem em risco sua sobrevivência. São exemplos de aspectos-chave: ambiente externo (política, legislações, etc.); expectativas financeiras dos stakeholders; concorrentes; valores e princípios organizacionais; pontos fortes, fracos, ameaças e oportunidades; estratégias emergentes; etc. A seguir, analisam-se os indicadores e índices de desempenho da organização, os objetivos estratégicos e a missão e visão atuais da organização. A etapa anterior alimenta este processo. A partir desta análise da estratégia e dos aspectos-chave relacionados a ela, identificam-se e priorizam-se os fatores críticos de sucesso, os processos de negócio e as áreas funcionais críticas que são importantes para se atingir a estratégia formulada.

O próximo passo consiste na elaboração de um plano de divulgação, comunicação e implementação da estratégia para diferentes níveis hierárquicos da empresa. Para propor e priorizar ações de melhoria e mudanças, é necessário mapear os processos de negócio da organização, analisar diagnósticos dos processos, avaliar os indicadores de desempenho atuais. A etapa posterior consiste em gerenciar a implementação das ações de melhoria. Para isso, deve-se modelar e validar o processo de negócio futuro; planejar e detalhar as ações implementadas. Sendo assim, é necessário um sistema de medição de desempenho para suportar a transformação e avaliar o desempenho das ações de melhoria ao longo da mudança.

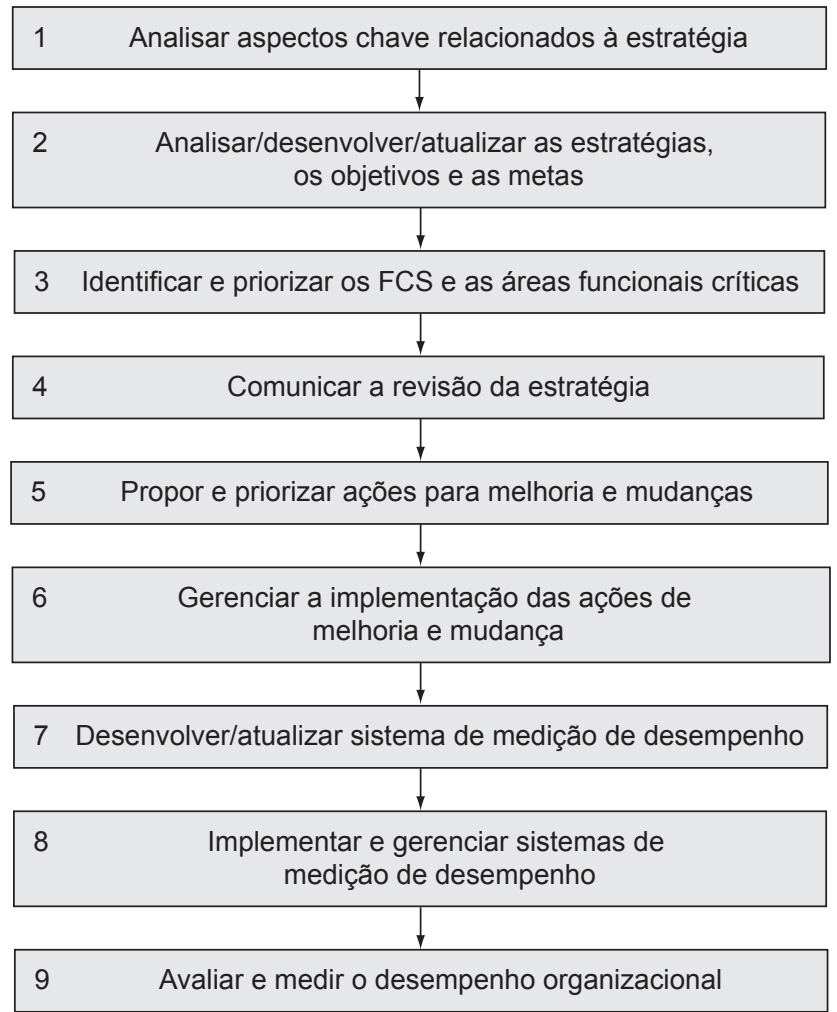

Figura 1. Proposta de sistematização para o processo de gestão de melhorias e mudanças de desempenho (GEROLAMO, 2003). 
Deve-se desenvolver e/ou atualizar o sistema de medição de desempenho. Deve-se identificar o sistema de medidas de desempenho atual da organização e sua relação com os fatores críticos de sucesso, a fim de avaliar o quanto o SMD está suportando os objetivos estratégicos da empresa.

Após esta avaliação, é preciso implementar e gerenciar o SMD. Por último, deve-se avaliar e medir o desempenho organizacional, no qual a empresa retrata seu desempenho.

\subsection{Desdobramento da estratégia}

Segundo este modelo, a primeira etapa para o desdobramento da estratégia é analisar e entender a missão da empresa. A partir deste entendimento, deve-se estabelecer uma estratégia compatível com a missão da empresa, isto é, deve-se estabelecer como a empresa irá cumprir sua missão. Após este passo, devem-se desenvolver metas estratégicas, ou seja, desmembrar a estratégia em metas e definir quais indicadores irão medir estas metas. Por fim, é necessário desenvolver ações para a concretização da estratégia e, conseqüentemente, o alcance das metas estratégicas (Figura 2).

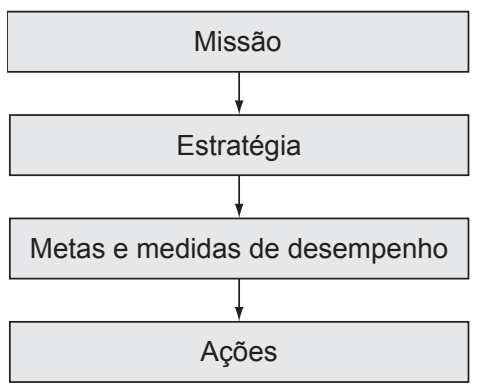

Figura 2. Modelo de planejamento estratégico.

\subsection{Balanced scorecard}

Segundo Niven (2002), pode-se descrever o BSC como um conjunto cuidadosamente selecionado de medidas derivadas da estratégia. Essas medidas representam um instrumento essencial para os gestores utilizarem na comunicação dos resultados e do comportamento dos direcionadores de desempenho para os empregados e acionistas das organizações, contribuindo para que elas atinjam sua missão e objetivos estratégicos.

Kaplan e Norton (2002) definem o balanced scorecard da seguinte forma:

[...] o "balanced scorecard" - conjunto de indicadores que proporciona aos gestores uma visão rápida, embora abrangente, de toda a empresa. $\mathrm{O}$ balanced scorecard inclui indicadores financeiros, que mostram o resultado das ações do passado, e os complementa com indicadores operacionais, relacionados com a satisfação dos clientes, com os processos internos e com a capacidade da organização de aprender e melhorar - atividades que impulsionam o desempenho financeiro futuro. (KAPLAN; NORTON, 2002).

Niven (2002) ressalta que o problema fundamental não é o desenvolvimento de uma estratégia e, sim, sua implementação através da tradução dessa estratégia em termos que facilitem o entendimento de todos os componentes da organização, os quais poderão direcionar suas ações individuais para o alcance dos objetivos organizacionais. Assim, o balanced scorecard constitui-se em um método de gestão estratégica, que pode ser utilizado para administrar a estratégia a longo prazo, viabilizando processos gerenciais críticos que se interligam (conforme a Figura 3), como:

a) traduzir a estratégia da unidade de negócios em objetivos estratégicos específicos;

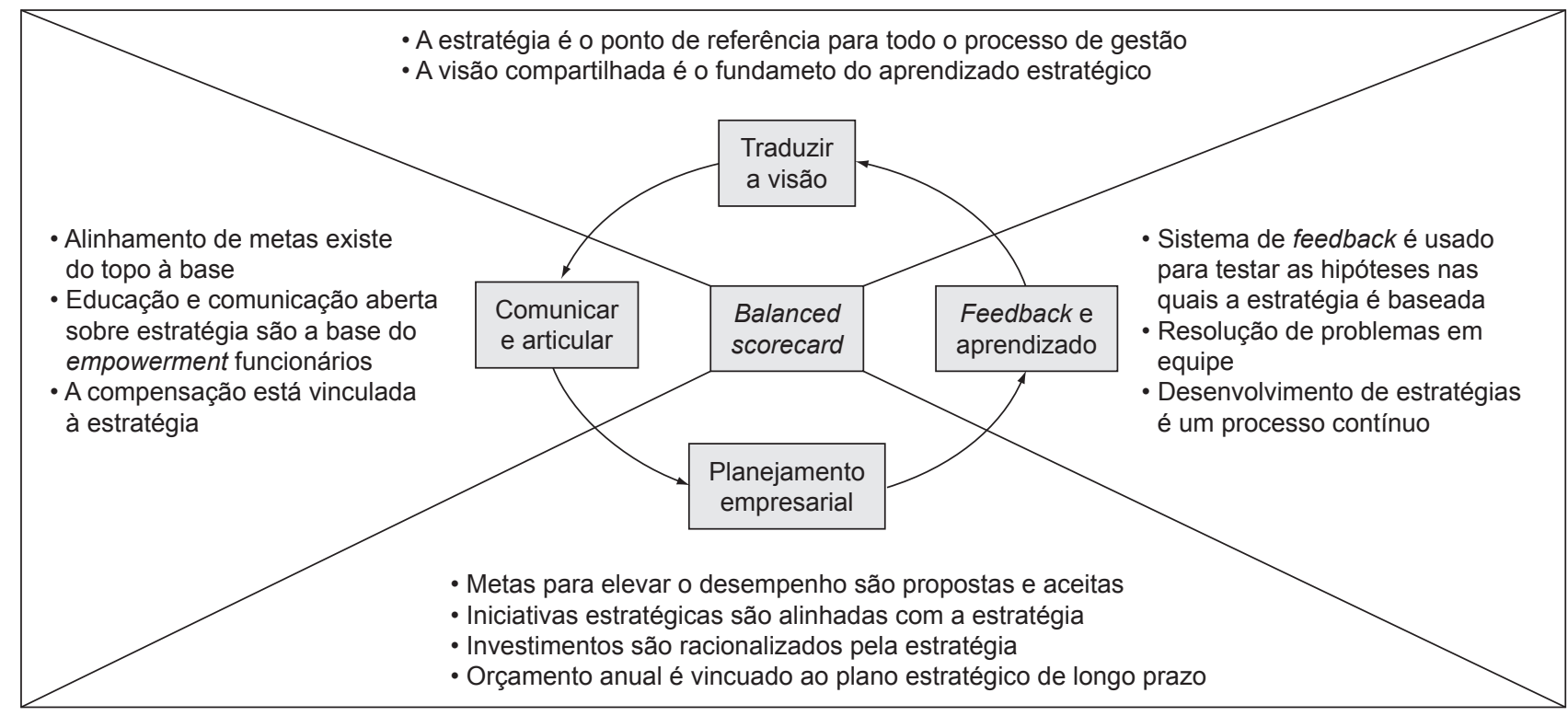

Figura 3. O BSC como estrutura para ação estratégica (KAPLAN et al., 2002). 
b) comunicar os objetivos e medidas estratégicas à empresa como um todo;

c) estabelecer metas;

d) alinhar iniciativas estratégicas (de qualidade) para objetivos extraordinários; e

e) melhorar feedback e aprendizado estratégico.

\subsection{Processo de seleção de projetos Seis Sigma}

De acordo com Pyzdek (2003), Arthur (2000), Adams et al. (2003), Basu (2003), Pande et al. (2001), Rotandaro (2002), Eckes (2001) e Snee e Rodenbaugh (2002), a seleção de projetos Seis Sigma é um fator crítico para o sucesso do programa Seis Sigma, pois projetos bem selecionados contribuirão para o sucesso e a consolidação da cultura Seis Sigma na empresa. A seguir, demonstra-se, na Figura 4, o processo de seleção de projetos segundo Werkema (2004).

A primeira etapa do processo de seleção de projetos Seis Sigma consiste na determinação dos objetivos estratégicos determinados por parte da alta gerência. Os projetos deverão contribuir para o alcance de pelo menos um desses objetivos. Logo após, deverá ser estabelecida uma relação de potenciais projetos Seis Sigma.

$\mathrm{Na}$ etapa de selecão de potenciais projetos, a equipe diretora realiza brainstorms com o objetivo de selecionar projetos de acordo com os problemas atuais da empresa.

Determinar os objetivos estratégicos da empresa

Estabelecer uma relação dos potenciais projetos Seis Sigma

Elaborar a matriz de priorização em relação aos objetivos estratégicos

Elaborar a matriz priorização em relação aos critérios $\downarrow$

Elaborar a matriz priorização em relação aos principais projetos

Selecionar os projetos que serão executados

Definir os responsáveis por cada projeto

Definir a equipe que trabalhará no projeto

Elaborar o Business Case

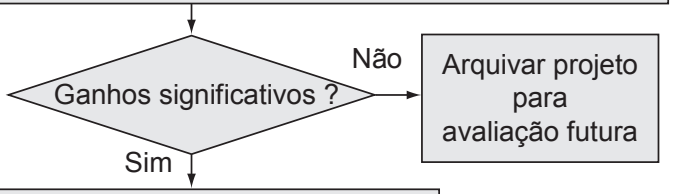

Indicar o desenvolvimento do projeto

Figura 4. Processo para seleção de projetos Seis Sigma (WERKEMA, 2004).
Estabelecidos os projetos potenciais, a próxima etapa é elaborar e aplicar a matriz de priorização para a avaliação do impacto dos potenciais projetos sobre os objetivos estratégicos (PATTTERSON; BERTELS, 2003). Um exemplo desta matriz pode ser observado na Tabela 1. Para a avaliação do impacto dos potenciais projetos sobre os objetivos estratégicos, primeiramente, deve-se ponderar a intensidade com que cada objetivo estratégico é relacionado com o projeto. Sendo assim, estabelecese uma escala de 0 a 5 , na qual 0 significa que não há relacionamento entre o projeto e o objetivo estratégico; 1 significa que o relacionamento é fraco; 3 , que o relacionamento é moderado; e 5, que o relacionamento é forte.

Para cada projeto, multiplicar o número resultante da intensidade do relacionamento pelo grau de importância do objetivo estratégico correspondente e somar os resultados das multiplicações. O resultado deve ser alocado na coluna "Impacto estratégico", na linha correspondente ao projeto diagnosticado. Cada soma deve ser transformada em um número na escala de 0-1-3-5 e registrada na coluna "Contribuição para o alcance dos objetivos estratégicos". Por fim, para cada potencial projeto, identificar a duração médio prazo (MP) ou longo prazo (LP).

A quarta etapa consiste em elaborar a matriz de priorização para seleção de projetos a partir de critérios para definição de um bom projeto Seis Sigma.

O método para elaborar a matriz de priorização é o mesmo descrito para a matriz anterior, porém cada coluna da matriz de priorização para seleção de projetos apresenta um critério ou filtro que a empresa utiliza para definir um bom projeto Seis Sigma. O grau de importância atribuído a cada critério (escala de 5 a 10) é uma conseqüência das estratégias da empresa. Assim, para cada projeto listado, identificar a intensidade com que cada critério é atendido, utilizando a mesma escala da matriz priorização anterior. Para cada projeto, multiplicar o número resultante da etapa anterior pelo grau de importância de critério correspondente e somar os resultados das multiplicações, registrando este resultado na coluna

"Total", na linha correspondente ao projeto. Quanto maior for o número na coluna "Total", maior será a prioridade do projeto como um projeto Seis Sigma.

\subsection{Proposta de um modelo para alinhamento estratégico de projetos Seis Sigma}

Segundo Santos e Martins (2005), o programa Seis Sigma vem ganhando força nas organizações como um programa de qualidade que promove a elevação do desempenho organizacional e, por isso, está cada vez mais inserido no plano estratégico das organizações, estabelecendo uma ligação com a infra-estrutura que a organização dispõe para medir o desempenho. O que, muitas vezes, tem como resultado um mau aproveitamento do programa, já que todo e qualquer problema que venha a surgir vira 
Tabela 1. Matriz prioridade para avaliação da relação entre o objetivo estratégico e os projetos (WERKEMA, 2004).

\begin{tabular}{|c|c|c|c|c|c|c|c|}
\hline $\begin{array}{l}\text { Legenda } \\
\text { O objetivo é : } \\
0 \text { - Não é relacionado; } \\
1 \text { - Fracamente relacionado; } \\
3 \text { - Moderadamente relacionado; } \\
5 \text { - Fortemente relacionado }\end{array}$ & 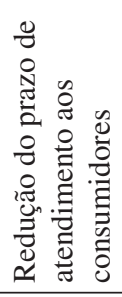 & 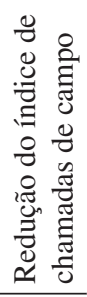 & 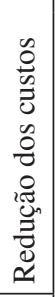 & \multirow{4}{*}{ 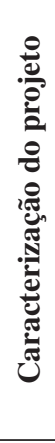 } & \multirow{4}{*}{ 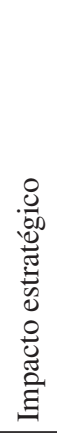 } & \multirow{4}{*}{ 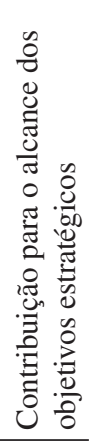 } & \multirow{4}{*}{$\begin{array}{l}0 \\
\text { త్తి } \\
1\end{array}$} \\
\hline Número do objetivo & 1 & 2 & 3 & & & & \\
\hline Peso para cada objetivo (5 a 10) & \multirow[t]{2}{*}{10} & \multirow[t]{2}{*}{7} & \multirow[t]{2}{*}{6} & & & & \\
\hline Potenciais projetos & & & & & & & \\
\hline Reduzir em $50 \%$ as devoluções dos clientes por problemas na embalagem, até 30/10/05 & 1 & 5 & 3 & & 63 & 3 & MP \\
\hline Reduzir em $70 \%$ o índice de anomalias nos motores importados até 31/12/05 & 3 & 5 & 3 & & 83 & 5 & LP \\
\hline Reduzir em $30 \%$ o custo de material comprado, até 21/12/05 & 0 & 0 & 5 & & 30 & 1 & $\mathrm{MP}$ \\
\hline
\end{tabular}

um projeto Seis Sigma, problemas estes que poderiam ser resolvidos com uma metodologia mais simples.

Selecionar corretamente os processos críticos carentes de melhoria, para que se alcance a elevação de desempenho organizacional, é um dos desafios primários do Seis Sigma (SANTOS; MARTINS, 2005). Como o alinhamento é um fator-chave para a consistência de propósitos e ações diante das iniciativas de melhoria, o programa Seis Sigma é um catalisador desse alinhamento por ser um programa estratégico que promove a melhoria de todo o negócio a partir do alcance dos objetivos estratégicos.

O processo de seleção e priorização de projetos Seis Sigma está diretamente relacionado ao alinhamento, isto é, a seleção de projetos dirige a força de melhoria para os processos críticos da empresa.

Santos e Martins (2005) propõem uma estrutura que expressa a relação dos indicadores de desempenho com o programa Seis Sigma, como reflexo do alinhamento estratégico. A estrutura é descrita sob nove pontos:

a) A organização interage com o ambiente para definição da estratégia;

b) Construção do sistema de medição de desempenho que esteja alinhado à estratégia;

c) As múltiplas categorias do sistema de medição de desempenho direcionam ações do programa Seis Sigma por meio do alinhamento na seleção de projetos Seis Sigma;

d) Os projetos Seis Sigma devem estar alinhados com a estratégia por meio de seus objetivos;

e) Os resultados oriundos da implantação dos projetos Seis Sigma podem promover a melhoria da eficiência e eficácia dos processos da empresa;

f) Os projetos Seis Sigma podem precisar de revisão dos seus objetivos para assim alcançar um maior alinhamento;

g) Os projetos Seis Sigma têm um papel de direcionadores de desempenho pela ligação que estabelecem com as medidas de desempenho que compõem o sistema de medição de desempenho;

h) A satisfação dos clientes é alcançada à medida que bens e serviços são melhorados; e

i) O sucesso da implementação de projetos Seis Sigma possibilita um feedback estratégico importante para a revisão dos objetivos estratégicos.

Pode-se perceber nesta estrutura que a seleção de projetos tem uma forte relação com os indicadores e com o direcionamento estratégico da empresa, sendo os pontos três e quatro desta estrutura, listados anteriormente. Esta sistematização, porém, não descreve com detalhes o processo de seleção de projetos Seis Sigma, contudo detalhou o desdobramento da estratégia e a importância do seu alinhamento com os projetos Seis Sigma, por meio do processo de seleção de projetos Seis Sigma.

\subsection{Consideração sobre as abordagens}

Segundo Gerolamo (2003), embora a questão estratégia seja complexa e dependa de inúmeras áreas e de uma dispersa fonte literária, é importante que as organizações busquem métodos para auxiliar em seus processos de análise, formulação, desenvolvimento e revisão da estratégia. Assim, as metas e os objetivos estratégicos terão maior probabilidade de serem alcançados se a estratégia direcionar a força de melhoria, e a força de melhoria para este artigo são os projetos Seis Sigma.

Analisando as abordagens propostas sobre desdobramento da estratégia e alinhamento estratégico, percebe-se que a abordagem de Kaplan e Norton (1990) descreve o desdobramento da estratégia, mas não detalha o alinhamento com a força de melhoria, enquanto que a abordagem de Gerolamo (2003) descreve com detalhes o alinhamento da estratégia com a força de melhoria sem analisar o desdobramento da estratégia. A abordagem de Yip (1995) é simples e sem muitos detalhes. No entanto, estes autores retratam a importância de se ter um SMD 
que motive melhorias em áreas críticas, o que a autora Werkema (2004) não deixa claro sobre a utilização destes na seleção de projetos Seis Sigma.

Desta forma, justifica-se um método sistemático que apresente com detalhes o alinhamento da estratégia com os projetos Seis Sigma.

\section{Proposta de sistematização do alinha- mento estratégico de projetos Seis Sigma}

Esta proposta aqui apresentada está embasada nas abordagens apresentadas no referencial teórico, são elas: Gerolamo (2003); Werkema (2004); Kaplan e Norton (1990); Yip (1995); e Santos e Martins (2005). A primeira abordagem citada apresenta um modelo conceitual sobre o processo de gestão de melhoria e mudança de desempenho de uma forma geral, faltando uma contextualização no programa Seis Sigma. Já a abordagem de Werkema (2004) não detalha o processo de alinhamento, mas destaca a sua importância, apresentando, assim, uma lacuna, que é o foco desta pesquisa. Kaplan e Norton (1990) deixam claro o desdobramento da estratégia, mas não explicitam seu alinhamento com a força de melhoria e, como dito anteriormente, Yip (1995) traz uma abordagem sem muitos detalhes. Assim, a maior motivação desta proposta é juntar e/ou adaptar as abordagens citadas preenchendo as lacunas supracitadas, sendo este um diferencial desta proposta, pois considera a sistematização do alinhamento da estratégia (o que as proposta de Werkema (2004) e Yip (1995) não apresentam) dos projetos Seis Sigma (o que as abordagens de Gerolamo (2003) e Kaplan e Norton (1990) não contextualizam especificadamente, levando em consideração a importância da seleção de projetos Seis Sigma). A proposta de Santos e Martins (2005) ressalta a importância do uso de indicadores de desempenho, mas não detalham o processo de seleção de projetos Seis Sigma. A Figura 5 ilustra a proposta de sistematização.

Cabe ressaltar que os passos um, dois e três são básicos para a implantação de um programa de qualidade como o Seis Sigma, isto é, o Seis Sigma só terá sucesso se for implantado em uma empresa com um mínimo de organização e maturidade em relação ao desdobramento da estratégia, por isso, estes passos irão focar o resgate e a atualização dos conceitos apresentados e não seu desenvolvimento.

Faz-se necessário ressaltar que esta pesquisa não irá detalhar o processo de definição da estratégia, pois este processo não faz parte do escopo deste trabalho.

\subsection{Resgate/atualização da missão da organização}

O primeiro passo da sistematização proposta é fazer um resgate da missão da organização. Neste processo, deve-se rever a missão da empresa e atualizá-la.

Muitos entendem mal a natureza e importância da missão, enquanto outros nem sequer a consideram. Drucker

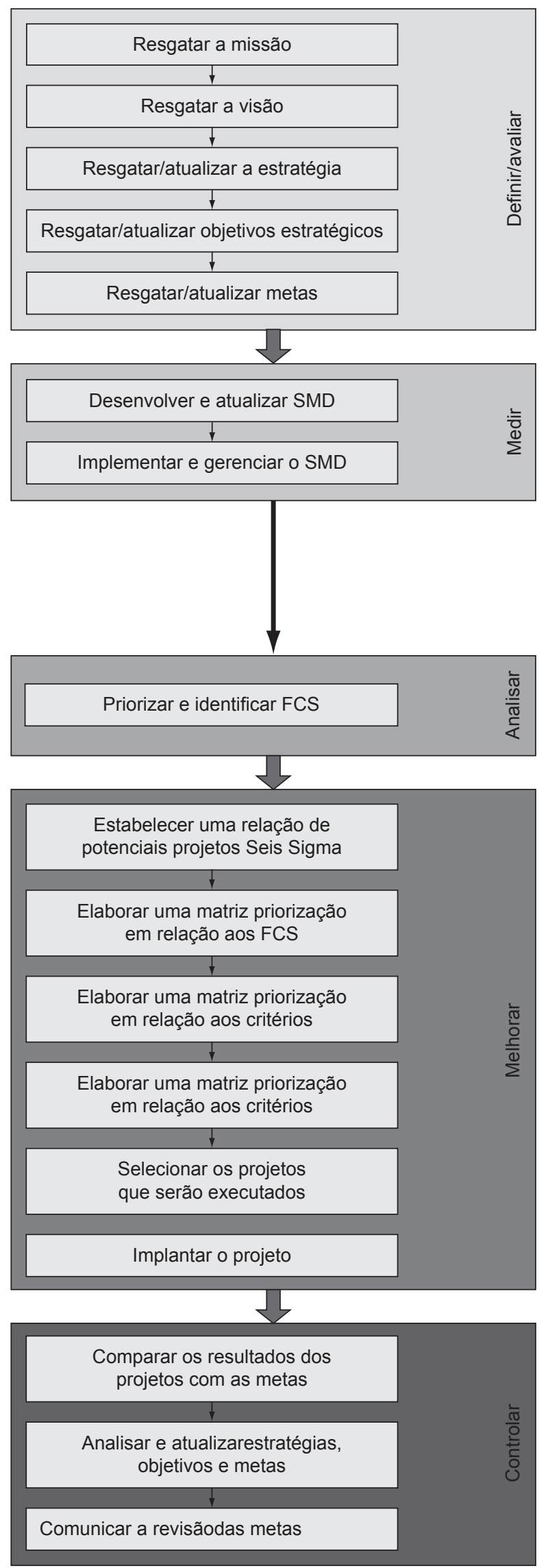

Figura 5. Proposta da pesquisa. 
(1973) observou: “A finalidade e a missão da empresa são tão raramente consideradas, que talvez essa seja a principal causa da frustração e fracasso das empresas".

A missão de uma organização pode ser definida como sua função principal, preferencialmente relatada em uma frase, deixando claro por que a organização existe (CHIAVENATO, 2004).

Os objetivos essenciais do negócio estão envolvidos na missão da organização. A missão é a razão de ser de uma organização, na qual se procura determinar seu negócio. E cada organização tem sua missão específica, da qual decorrem seus objetivos organizacionais principais (CHIAVENATO, 2004).

\subsection{Resgate/atualização da visão e estratégia}

Nesta fase, a organização deve rever sua estratégia para resgatar sua visão, isto é, a organização deve analisá-la e atualizá-la para que ela contemple a realidade atual da empresa.

Deve-se obter um conjunto de princípios e crenças que, juntamente com a declaração da missão, ajudará a alcançar essa visão (CHIAVENATO, 2004). Sendo assim, a visão é o estado futuro desejável, relacionado com a máxima satisfação dos clientes.

A partir do resgate da missão e da visão organizacional, o próximo passo é rever para resgatar a estratégia. Uma estratégia bem definida que retrate o atual contexto organizacional é necessária para que se tenha um programa Seis Sigma em andamento, pois este tipo de programa necessita de uma estrutura gerencial bem definida.

\subsection{Resgate/atualização dos objetivos estraté- gicos e das metas da organização}

A estratégia deve ser desdobrada em objetivos estratégicos, isto é, as organizações são entidades orientadas para objetivos. Os objetivos estratégicos são objetivos globais e amplos da organização. Em alguns casos, os objetivos estratégicos são desdobrados em objetivos táticos e operacionais ou em metas. (CHIANENATO, 2004). Estes objetivos devem ser sempre revisados para se obter o desempenho da organização em relação aos objetivos estipulados; nesta fase se realizará esta revisão.

Uma meta, qualquer que seja ela, só pode ser assim conceituada quando traçada segundo algumas variáveis (CAMPOS, 2002). Seu objetivo deve ser muito bem definido, pois quanto mais específica for a definição de seu propósito, mais direcionado estará seu caminho.

A meta deve ser quantificável, tornando-se objetiva, palpável. Uma meta tem que ser alcançável, possível, viável. Além disso, a meta tem que ser importante, significativa, desafiadora. Finalmente, as metas devem ser bem definidas, mensuráveis, possíveis, importantes e definidas num horizonte de tempo.
É necessário resgatar as metas, verificar quais foram alcançadas e quais terão que ser alcançadas, e as que não foram alcançadas podem ser os objetivos de um projeto Seis Sigma, já que a meta surgiu do desdobramento da estratégia.

\subsection{Desenvolver, atualizar e implementar o Sistema de Medição de Desempenho}

Fazendo uma revisão dos passos anteriores, pode-se afirmar que determinar a direção estratégica significa criar a missão e definir os objetivos estratégicos, levando em consideração a visão e os valores da organização. Além disso, a direção estratégica está relacionada a escolher o destino e o caminho corretos para a organização. Essa escolha requer um alto grau de compreensão do ambiente externo bem como uma avaliação correta das capacidades e competências da organização (GEROLAMO, 2003).

Uma vez escolhida a estratégia, é necessário escolher as metas e os indicadores de desempenho que mostrarão se a organização está no caminho planejado e, por meio de uma avaliação, o quanto de progresso foi feito na direção dos objetivos estratégicos. Sendo assim, o próximo passo é desenvolver e atualizar o sistema de medição de desempenho. Nesta fase, deve-se identificar o sistema de medidas de desempenho, as quais irão mensurar quanto a organização está ou não alcançando suas metas. É necessário desenvolver e atualizar o Sistema de Medição de Desempenho (SMD) para que este esteja apoiando os objetivos estratégicos da empresa. Para tanto, deve-se identificar o relacionamento entre os indicadores, definir as medidas das metas, identificar os indicadores conflitantes, detalhar os indicadores.

Nesta fase, deve-se operacionalizar o SMD, buscando sempre validar a operacionalização e avaliando periodicamente o SMD.

\subsection{Priorizar e identificar o Fator Crítico para o Sucesso}

Segundo Chiavenato (2004), os fatores críticos de sucesso (FCS) são os elementos condicionantes no alcance dos objetivos da organização e estão ligados diretamente com o sucesso da empresa. Para identificar os FCS, existem duas maneiras. A primeira é dissecar os recursos organizacionais e o mercado a fim de identificar os segmentos que são mais decisivos e importantes. A segunda é descobrir o que distingue as organizações mal sucedidas das organizações bem sucedidas e analisar a diferença entre elas, isto é, fazer um benchmarking (CHIAVENATO, 2004).

Após a identificação, os FCS devem ser priorizados. Para priorizar, deve-se analisar, por meio do SMD, em quais FCS a organização está mal sucedida e focar a força de melhoria neles (BROWN, 2000). 


\subsection{Estabelecer uma relação de potenciais pro- jetos Seis Sigma}

Tendo já identificado e priorizado os FCS, deve-se estabelecer uma lista de possíveis projetos Seis Sigma focados nos FCS priorizados anteriormente, isto é, os projetos Seis Sigma de melhoria devem solucionar os problemas das FCS.

\subsection{Elaborar uma matriz de priorização em rela- ção aos FCS}

Esta matriz é uma ferramenta para relacionar o projeto com os objetivos estratégicos da empresa.

\subsection{Elaborar uma matriz de priorização em rela- ção aos critérios}

Esta matriz pretende relacionar os projetos que passaram pelo filtro da matriz anterior com outros critérios. Tais critérios são características importantes que os projetos têm que ter para serem selecionados, como prazo de execução do projeto, facilidade dos dados, custo do projeto, aumento da satisfação do cliente, disponibilidade da equipe envolvida, retorno financeiro dos projetos, etc.

\subsection{Selecionar os projetos que serão executados}

Nesta fase, os projetos que passaram pelos filtros das matrizes anteriores serão selecionados para sua execução.

\subsection{Implantar o projeto}

Aqui, desenvolver-se-á o projeto baseado na metodologia DMAIC, a qual não é o foco desta pesquisa.

\subsection{Comparar os resultados dos projetos com as metas pretendidas}

Pretende-se, nesta fase, comparar os resultados realmente alcançados com os projetos com os resultados esperados na fase de planejamento e seleção de projetos Seis Sigma. Sendo assim, esta fase realizará um monitoramento do desempenho dos projetos e dos processos de seleção de projetos, ou seja, se as metas e objetivos da organização foram alcançados por meio dos resultados dos projetos, isto significa que os projetos foram bem selecionados e estão realmente alinhados à estratégia da empresa.

O monitoramento do desempenho é o processo administrativo que garante que aquilo que uma organização está fazendo está consistente com o que ela decidiu fazer.

Os indicadores de desempenho que constituem o monitoramento de desempenho devem garantir este processo de monitoramento.

\subsection{Analisar e atualizar estratégias, objetivos, metas e comunicação}

Nesta fase, devem ser analisados os indicadores e índices de desempenho da organização, a estratégia, a missão, visão, objetivos e metas atuais da organização.
Os objetivos e metas já alcançados devem ser substituídos ou atualizados de acordo com os novos resultados obtidos com os projetos Seis Sigma.

O último passo consiste na elaboração de um plano de divulgação, comunicação das novas metas e objetivos determinados na etapa anterior.

\section{Estudo de caso}

\subsection{América Latina Logística}

A América Latina Logística (ALL) oferece serviços de logística de grande porte, com operações de transporte "porta-a-porta" intermodal, doméstico e internacional, distribuição urbana, serviços de terminal portuário, frota dedicada, serviços de armazenamento, incluindo a gestão de estoques e centros de distribuição.

O alinhamento dos projetos Seis Sigma na ALL é uma responsabilidade da diretoria e é feito por meio da seleção de projetos Seis Sigma. Presidente e diretores analisam os pontos fracos que a ALL possui hoje por meio de um sistema de medição de desempenho que aponta vermelho para indicadores críticos, amarelo para indicadores que podem se tornar críticos em um futuro bem próximo e verde para indicadores que estão dentro da meta proposta pela empresa. É importante ressaltar que este sistema de medição de desempenho da empresa mede o desempenho em relação à estratégia adotada pela empresa, isto é, ele está diretamente interligado com a estratégia.

Tendo o conhecimento dos indicadores críticos, ou seja, os vermelhos, aqueles cujas metas propostas a companhia ainda não conseguiu atingir, os projetos Seis Sigma são direcionados a estes indicadores para que se tornem verdes. Dentro das áreas críticas, são apontados alguns projetos pela diretoria. Dentro dos projetos propostos são escolhidos os colaboradores que irão desenvolver os projetos, os quais passam por uma nova avaliação que contempla alguns requisitos que os projetos devem apresentar, como: tempo máximo de execução de um ano, custo de no máximo $30 \%$ do ganho estimado do projeto, ter um colaborador já envolvido nesta área para oferecer treinamento e o que está dentro da estratégia da empresa naquele momento.

Os selecionados para desenvolver projetos Seis Sigma passam a ter uma relação direta com os diretores da empresa, que fiscalizam a operacionalização dos projetos. Os melhores projetos green belt são premiados e validados pela empresa Wekema Consultoria, e os colaboradores responsáveis recebem treinamento para black belt. Normalmente são treinados cinco green belts por ano. O projeto do black belt é um aprofundamento do projeto de green belt na mesma linha do projeto anterior. Sendo assim, há uma remuneração variável que é oferecida no fim do ano em que é traçada uma meta por colaborador até certo nível da empresa, que, no caso de 
green belts e black belts, é maior e atrelada aos resultados dos projetos desenvolvidos.

A proposta apresentada por este artigo é semelhante ao processo de alinhamento da estratégia com projetos Seis Sigma desenvolvido na empresa. Isso porque o processo de alinhamento da empresa possui as etapas: definir, medir, analisar, melhorar e controlar. E, após a apresentação, os entrevistados reconheceram as semelhanças, como por exemplo: de início deve-se começar pela análise da estratégia, a necessidade de um sistema de medição de desempenho que aponte as áreas críticas ajustado à estratégia e, a partir de então, selecionar os projetos direcionados a estas áreas.

A ALL afirma ter tido problemas com projetos que não atingiram a meta estabelecida ou não se desenvolveram no prazo determinado. A causa principal não é um processo de seleção de projetos mal direcionado ou mal desenvolvido e sim o fato das pessoas envolvidas não terem tempo disponível somente para realizarem os projetos, tendo que, além de fazerem as tarefas do dia-a-dia, alcançarem as metas dos projetos Seis Sigma. Isso significa que a empresa se encontra satisfeita com os resultados do processo de alinhamento da estratégia com projetos Seis Sigma.

A empresa relatou que o estabelecimento de um modelo formal para este processo pode melhorar o alinhamento da estratégia com projetos Seis Sigma e que não teria dificuldade nenhuma em adotar a proposta desta pesquisa, pois já está inserida na cultura da empresa a metodologia DMAIC e, principalmente, a medição de desempenho que teve seu início bem antes do programa Seis Sigma, com o gerenciamento pelas diretrizes. A empresa, perante o modelo proposto, não mudaria nenhuma etapa e não adicionaria nenhum passo, pois este se assemelha ao modelo existente e traz resultados satisfatórios.

\subsection{Companhia Vale do Rio Doce}

Os serviços de logística oferecidos pela Companhia Vale do Rio Doce (CVRD) incluem diagnóstico, modelagem e implementação de operações logísticas completas. A área de Logística é suportada por 9.306 km de malha ferroviária e dez terminais portuários próprios, que oferecem soluções para a gestão logística e o transporte de mercadorias porta-a-porta, complementando operações com a utilização intensa do modal rodoviário.

O programa Seis Sigma na CVRD hoje é a maior e melhor ferramenta de soluções de problemas existentes na empresa segundo o entrevistado. O programa teve início em janeiro de 2004, quando foram treinados 470 colaboradores de todas as unidades.

$\mathrm{Na}$ CVRD, o projeto de melhoria é um pré-requisito para o treinamento, isto é, recebe o treinamento aquele colaborador que apresentar um projeto de melhoria. Portanto, não são todos os colaboradores que podem escrever projetos em suas áreas e que receberão trei- namento, somente aqueles que apresentam projetos relacionados às metas estratégicas da empresa.

O entrevistado considera que o programa Seis Sigma é formado por projetos de melhoria drástica e se esta melhoria não estiver vinculada às metas da organização, não fará sentido o projeto Seis Sigma.

A Diretoria de Gestão de Desempenho detecta as necessidades da organização pelo sistema de medição de desempenho, alertando para as áreas que mais necessitam de projetos e traçando as metas. A partir de então, os colaboradores envolvidos nas áreas escrevem o projeto e ele é analisado pela mesma diretoria, que faz a avaliação e a seleção de projetos.

Esta avaliação é feita principalmente sob um critério: o alinhamento da estratégia com o projeto, isto é, quanto mais o projeto contribuir com as metas da organização, mais chances ele tem de ser o escolhido, reconhecendo que o alinhamento da estratégia com projetos Seis Sigma é crítico para o sucesso do programa.

Após a avaliação da proposta de sistematização do alinhamento da estratégia com projetos Seis Sigma, o entrevistado realizou algumas observações. A primeira foi ressaltar a importância deste alinhamento da estratégia e as conseqüências que a organização pode ter se não tiver projetos Seis Sigma comprometidos com suas metas. A segunda, analisando a proposta, o entrevistado sugeriu uma mudança de termos, a substituição do termo revisão nos cinco primeiros passos para conhecer, pois, no seu entendimento, revisar pode sugerir a idéia de fazer de novo ou atualizar, o que não compete aos envolvidos no programa Seis Sigma.

Outra observação é que esta sistematização deve ser top down, isto é, as decisões devem partir da alta gerência. Além disso, os problemas na seleção de projetos Seis Sigma quase sempre são oriundos do processo de estabelecimento de metas e desdobramento da estratégia.

Portanto, o entrevistado concorda com a proposta apresentada, em vista das observações feitas.

\subsection{MasterDis}

A MasterDis é uma grande empresa distribuidoraatacadista, sendo uma das maiores da América Latina. $\mathrm{Na}$ central de distribuição, a mercadoria é alojada segundo um mapa que divide as prateleiras em quarteirões, avenidas, ruas e apartamentos.

A MasterDis é um nome fantasia atribuído a um dos casos, pois o nome real não pode ser divulgado segundo a empresa pesquisada. Esta empresa é operacionalizada pela Divisão de Logística, que armazena, movimenta, fraciona, aglutina, transporta e distribui os produtos.

$\mathrm{Na}$ empresa, já foram encerrados aproximadamente cem projetos Seis Sigma. Na MasterDis, segundo o entrevistado, não há um método bem estruturado para a seleção de projetos Seis Sigma, pois o programa foi paralisado 
em 2004. Nem mesmo o entrevistado conseguiu identificar o relacionamento entre os projetos e a estratégia da empresa, apontando que este pode ser um dos motivos do não comprometimento da alta gerência de projetos, pois faltam objetivos claros e concisos com a realidade da empresa, resultando na paralisação do projeto, como aconteceu em 2004.

$\mathrm{Na}$ MasterDis, de acordo com o entrevistado, normalmente não há uma seleção de projetos e sim a identificação de que este é necessário pela alta diretoria e que trará redução de custos. Assim sendo, um green belt ou black belt é alocado para conduzir o projeto.

Há, na empresa, o desdobramento da estratégia e a identificação das áreas críticas para o sucesso, porém nem sempre os projetos são alocados nestas áreas, pois deveria haver o comprometimento do gerente da área, o que nem sempre ocorre.

Outros critérios que influenciam na seleção de projeto Seis Sigma na MasterDis são principalmente retorno financeiro (quanto maior melhor) e disponibilidade operacional, isto é, se existe mão-de-obra qualificada para o desenvolvimento do projeto.

A MasterDis, segundo a análise das respostas do entrevistado, não possui um método sistemático para o alinhamento da estratégia dos projetos Seis Sigma, o que foi apontado pela própria empresa como uma dificuldade do programa.

Após a apresentação da proposta de sistematização desta pesquisa, o coordenador do programa Seis Sigma apresentou algumas dificuldades em adotá-la: a MasterDis não apresenta um sistema de desdobramento da estratégia em metas como foi proposto e não possui um sistema de medição de desempenho que auxilie o processo de alinhamento da estratégia dos projetos.

A empresa ressalta, porém, que não é a proposta apresentada que deve ser alterada, e sim a empresa que deve buscar este desdobramento e ajustar o sistema de medição de desempenho ao programa Seis Sigma, concluindo que a proposta, na opinião da empresa, parece ser eficaz e pode gerar melhores resultados do que a forma atual que a empresa possui de selecionar projetos Seis Sigma.

Na verdade, não existe um sistema de metas já estabelecidas, existem metas por departamento e os projetos Seis Sigma não estão vinculados. Os colaboradores selecionados para realizar o treinamento deverão fazer um projeto em sua respectiva área, não importando se esta é crítica ou não.

$\mathrm{Na}$ empresa, esforços e capital são investidos em projetos que não fazem parte da estratégia da empresa, o que ocasionou uma desmotivação em relação ao programa Seis Sigma e, posteriormente, sua paralisação em 2004. Ainda que, de acordo com a análise de documentos apresentados pela empresa, muito capital foi investido, ou seja, cerca de $\mathrm{R} \$ 415.310$, por outro lado, a empresa obteve muito lucro com o programa, cerca de 27 milhões. Logo, como o programa gerou muitos benefícios, houve um retorno ao programa em 2005, mas ainda não possui um foco definido. Durante a visita às instalações, foi perguntado aos colaboradores que participam do programa, se eles sabiam da contribuição do projeto de melhoria Seis Sigma, que eles estavam desenvolvendo naquele momento para a estratégia da empresa. Muitos não souberam responder, e a empresa não permitiu divulgar números.

\subsection{Análise dos casos}

A análise dos casos apresentados permite que se destaquem alguns pontos, tais como: é imprescindível que a organização que esteja implantando o Seis Sigma seja madura em relação aos programas de qualidade e ao desdobramento da estratégia, pois este último é indispensável para o alinhamento da estratégia com projetos. De acordo com os casos apresentados, observa-se que nas organizações que antes do Seis Sigma já haviam implantado programas de qualidade tais como ISO 9000, gerenciamento pelas diretrizes, controle da qualidade, entre outros, o resultado do programa Seis Sigma foi mais satisfatório. É o caso da Vale do Rio Doce e da América Latina Logística.

Além disso, os conceitos missão, visão, metas e objetivos devem estar difundidos na organização, bem como já definidos e tendo origem na estratégia da empresa, visto que os projetos Seis Sigma, para estarem alinhados à estratégia, à missão, aos objetivos e metas, também devem estar alinhados à estratégia para que os projetos Seis Sigma passem a ser ferramentas para o alcance destes itens já citados.

Sabe-se também que, por intermédio das metas e objetivos derivados da estratégia, é possível determinar os fatores críticos para o sucesso (FCS) e priorizar os projetos que mais contribuirão para se atingir a estratégia.

Outro aspecto a ser observado é: apresentar um sistema de medição de desempenho que quantifique os objetivos e metas e que seja capaz de refletir a estratégia da empresa. Sendo assim, um sistema de indicadores de desempenho deve conter dados para monitorar o passado e planejar o futuro. Os indicadores de desempenho tradicionais e os não financeiros devem ser integrados dentro de um sistema único, no qual se devem considerar informações dos vários sistemas para fornecer o nível necessário de dados em termos de acuracidade e confiabilidade. Ao longo do tempo, o desenho de qualquer sistema de indicadores de desempenho deve refletir as operações básicas do suporte organizacional, sempre lembrando a importante relação intrínseca entre indicadores de desempenho e estratégia. Por meio do monitoramento de indicadores-chave é possível, por este sistema, identificar lacunas de desempenho e, então, sugerir projetos de melhoria Seis Sigma. 


\section{Conclusão}

Esta pesquisa apresentou a importância do alinhamento da estratégica com projetos Seis Sigma para o sucesso do programa, além de uma proposta de sistematização deste alinhamento com o objetivo de responder à seguinte questão: Qual é a importância de se alinhar o processo de seleção de projetos Seis Sigma à estratégia e como este alinhamento é alcançado?

Os casos estudados apontaram o que a literatura já tinha constatado, ou seja, a importância do alinhamento da estratégia com projetos Seis Sigma e a forma de se obter este alinhamento deve ser por meio dos passos da proposta apresentada, pois, nos casos em que se obtiveram sucesso neste alinhamento, os entrevistados identificaram os passos da proposta apresentada em suas respectivas empresas, considerando a proposta viável e útil, obtendo aqui uma relação entre os casos e a proposta apresentada.

Quanto à proposta de sistematização apresentada, deve-se ressaltar a importância de se ter na literatura uma sistematização do alinhamento da estratégia com projetos Seis Sigma, visto que, este alinhamento é essencial para o sucesso do programa. Além disso, a proposta contribui para as empresas que estejam implantando o programa Seis Sigma para que elas tenham sucesso na seleção dos projetos de melhoria.

A análise dos estudos de casos apresentados permitiu a apresentação da proposta de sistematização. Numa visão geral, a proposta apresentada não difere da realidade observada nas empresas Companhia Vale do Rio Doce e América Latina logística.
Já no caso da empresa MasterDis, porém, constatou-se a necessidade de alinhar os projetos Seis Sigma à estratégia da empresa, o que não estava ocorrendo de forma coesa, sendo este ponto apontado pelo entrevistado como crítico para o sucesso do programa que, em 2004, teve uma paralisação pela falta de objetivos claros e bem definidos.

Além disso, observou-se, por meio dos casos, que, quanto melhor estruturado e sistematizado o processo de seleção de projetos Seis Sigma, melhores são os resultados, pois o MasterDis que não possuía um método conciso para o alinhamento da estratégia com projetos Seis Sigma teve o programa estagnado por um ano dentro da empresa, enquanto as outras empresas colhiam resultados satisfatórios. Essa constatação foi a principal contribuição dos casos, pois, por meio da comparação entre as empresas, concluiu-se que a estruturação deste processo aumenta sua eficiência e eficácia.

Cabe ressaltar que, embora os casos desta pesquisa tenham sido operadores logísticos, a conclusão é que a importância do processo de alinhamento da estratégia com projetos Seis Sigma para o sucesso do programa não é restrito somente a este tipo de empresa, mas para todas as empresas que utilizam o programa Seis Sigma.

Não era o objetivo apresentar uma proposta abrangente para todas as ações de melhoria, o foco eram os projetos Seis Sigma. O alinhamento entre projetos Seis Sigma e a estratégia pode ser atingido por um processo sistematizado de alinhamento, que maximizará o sucesso do programa Seis Sigma, como o apresentado por esta pesquisa. Para pesquisas posteriores, recomenda-se a aplicação desta proposta apresentada.

\title{
The importance of the process of alignment the strategy with Six Sigma projects: a study multicases in logistic operator
}

\begin{abstract}
Besides being in evidence in the organizational environment, the Six Sigma programs and logistics' implementation has also been achieving success pointing out the importance of the strategy alignment of project improvements. Therefore, an analysis of the strategy alignment process with Six Sigma projects is necessary. Hence, this paper proposes a systematization of the alignment of the organizational strategy with the process of selecting Six Sigma projects. This study includes a brief description of the conceptual models used and the logistics operators. Based on theoretical considerations, a conceptual systematization of the alignment of the organizational strategy with a detailed process of selecting Six Sigma projects is proposed. Aiming at analyzing the conceptual proposal, three case studies in logistics operators were carried out. It can be said that the alignment of the process of selecting Six Sigma projects with the organizational strategy proved important and the systematization proposed proved efficient.
\end{abstract}

Keywords: Six Sigma projects. Strategy and alignment. 
ADAMS, C.; GUPTA, P.; WILSON, C. Six Sigma Deployment. New York: Butterworth Heinemann, 2003.

ANTONY, F. Some pros and cons of six sigma: an academic perspective. The TQM Magazine, v. 16, n. 4, p. 303-306, 2004.

ANTONY, J. Six Sigma in the UK service organizations: results from a pilot survey. Managerial Auditing Journal, v. 19, n. 8, p. 1006-1013, 2004.

ARTHUR, J. Six Sigma simplified: quantum improvement made easy. Denver: Lifestar, 2000.

BASU, R. Quality beyond six sigma. Oxford: Butterworth Heinemann, 2003.

BISGAARD, S.; HOERL, R.; SNEE, R. Improving business processes with Six Sigma. In: ANNUAL QUALITY CONGRESS, Milwaukee, 2002. Anais... Milwaukee, 2002. p. 701-704.

BOWERSOX, D. J.; CLOSS, D. J. Logística Organizacional: O Processo de Integração da Cadeia de Suprimento. São Paulo: Atlas, 2001.

BROWN, M. Winning score: how to design and implement winning scorecards. Portland, Oregon: Productivity Press, 2000.

BRYMAN, A. Research methods and organization studies. London: Unwin Hyman, 1989.

CAMPOS, V. Gerenciamento pelas diretrizes. Belo Horizonte: INDG, 2002.

CHIAVEnATO, I. Teorias da Administração. Rio de Janeiro: Campus, 2004.

DRUCKER, P. Management: Tasks, Responsabilities, Practices. New York: Harper \& Row, 1973. 78p.

ECKES, G. A revolução Seis Sigma: o método que levou a GE e outras empresas a transformar processos em lucro. Rio de Janeiro; Campus, 2001.

GEROLAMO, M. Proposta de sistematização para o processo de gestão de melhorias e mudanças de desempenho. São Carlos, 2003. 165f. Dissertação (Mestrado) - Escola de Engenharia de São Carlos, Universidade de São Paulo.
GIL, A. Como elaborar projetos de pesquisa. São Paulo: Atlas, 1993.

HARRY, M. Six sigma: the breakthrough Management strategy revolutionizing the world's top corporations. New York: Currency, 2000

HARRY, M.; CRAWFORD, D. Six Sigma - The next generation. Machine Design Cleveland, v. 77, n. 4, p. 126 -130, 2005.

HARRY, M.; SCHROEDER, R. Six Sigma: a breakthrough strategy for profitability. New York: Quality Progress, 1998.

KAPLAN, R. S.; NORTON, D. P. Organização orientada para a estratégia. Rio de Janeiro: Campus, 2002.

Organização orientada para a estratégia: como as empresas que adotaram o Balanced Scorecard prosperam. Rio de Janeiro: Campus, 1990.

NIVEN, P. R. Balanced scorecard step by step: maximizing performance and maintaining results. New York: John Wiley \& Sons, Inc, 2002.

PANDE P.; NEUMAN, R.; CAVANAGH, R. A estratégia Seis Sigma: como a Motorola e outras grandes empresas estão aguçando seu desempenho. Rio de Janeiro: Qualitymark, 2001.

PYZDEK, T. The six sigma project planner a step-by-step guide to leading a six sigma project through DMAIC. New York: McGraw-Hill, 2003.

ROTANDARO, R. Seis Sigma: estratégia gerencial para melhoria de processos, produtos e serviços. São Paulo: Atlas, 2002.

SANTOS, A.; MARTINS, M. Medição de desempenho e alinhamento estratégico: requisitos para o sucesso do programa Seis Sigma. In: SIMPÓSIO DE ADMINISTRAÇÃO DA PRODUÇÃO, LOGÍSTICA E OPERAÇÕES INTERNACIONAIS, 8., 2005, São Paulo. Anais..., São Paulo: FGV- EAESP, 2005.

SENAPATI, N. Six Sigma: myths and realities. International Journal of Quality \& Reliability Management, v. 21, n. 6, p. 683-690, 2004.

WERKEMA, C. Criando a cultura Seis Sigma. Nova Lima: Werkema, 2004.

\section{Sobre os autores}

\section{Bianca Soares de Oliveira Gonçalves}

Departamento de Engenharia de Produção, Universidade Federal de Goiás,

Campus Catalão, Av. Dr. Lamartine Pinto de Avelar, 1120, CEP 75700-000, St. Universitário, Catalão, GO, Brasil,

Programa de Pós-graduação de Engenharia de Produção,

Escola de Engenharia de São Carlos - EESC, Universidade de São Paulo - USP,

Av. Trabalhador São-carlense, 400, CEP 13566-590, Pq. Arnold Schimidt, São Carlos, SP, Brasil,

e-mail: opbianca@yahoo.com.br

\section{Marcel Andreotti Musetti}

Programa de Pós-graduação de Engenharia de Produção,

Escola de Engenharia de São Carlos - EESC, Universidade de São Paulo - USP,

Av. Trabalhador São-carlense, 400, CEP 13566-590, Pq. Arnold Schimidt, São Carlos, SP, Brasil,

e-mail: musetti@sc.usp.br 